\title{
Investigation of the dose- and time-dependence of the induction of different types of cell death in a small-cell lung cancer cell line: Implementation of the repairable-conditionally repairable model
}

\author{
NIKOS MAKRIS ${ }^{1}$, MARGARETA EDGREN ${ }^{2}$, PANAYIOTIS MAVROIDIS ${ }^{2,3}$ and BENGT K. LIND ${ }^{2}$ \\ ${ }^{1}$ Department of Medical Physics, Medical School, University of Patras, 26504 Patras, Greece; \\ ${ }^{2}$ Department of Oncology-Pathology, Division of Medical Radiation Physics, Karolinska Institutet, \\ 17176 Stockholm, Sweden; ${ }^{3}$ Department of Radiation Oncology, Division of Medical Physics, \\ University of Texas Health Sciences Center at San Antonio, San Antonio, TX 78229, USA
}

Received September 7, 2012; Accepted October 11, 2012

DOI: 10.3892/ijo.2013.1888

\begin{abstract}
The purpose of this study was to quantify and model various types of cell death for a small-cell lung cancer (SCLC) cell line (U1690) after exposure to a ${ }^{137} \mathrm{Cs}$ source and as well as to compare the linear-quadratic (LQ) and repairable-conditionally repairable model (RCR). This study is based on four different experiments that were taken place at Cancer Centrum Karolinska (CCK). A human small-cell lung cancer (SCLC) cell line after the exposure to a ${ }^{137} \mathrm{Cs}$ source was used for the extraction of the clonogenic cell survival curve. Additionally, for the determination and quantification of various modes of cell death the method of fluorescence staining was implemented, where the cell deaths were categorized based on morphological characteristics. The percentage of cells in each phase of the cell cycle was investigated with flow cytometry analysis. The quantification of senescent cells was performed by staining the samples with senescence-associated $\beta$-galactosidase (SA- $\beta$-Gal) solution and then scoring as senescent cells those that had incorporated the substance. These data were introduced into a maximum likelihood fitting to calculate the best estimates of the parameters used by the examined model. In this model, the modes of cell death are divided into three categories: apoptotic, senescent and other types of cell death (necrotic/apoptotic, necrotic, micronuclei and giant). In the clonogenic cell survival assay, the fitting of the RCR model gives a $\chi^{2}$-value of 6.10 whereas for the LQ model became 9.61. In the fluorescence microscopy and senescence assay, the probability of the three different modes of cell death on day 2 seems to increases with a dose up to about $10 \mathrm{~Gy}$ where there is saturation. On day 7 a significant induction of apoptosis in a
\end{abstract}

Correspondence to: Dr Panayiotis Mavroidis, Division of Medical Physics, Department of Radiation Oncology, University of Texas Health Sciences Center at San Antonio, 7979 Wurzbach Rd, MC 7889, San Antonio, TX 78229-4427, USA

E-mail:mavroidis@uthscsa.edu

Key words: small cell lung cancer, repairable-conditionally repairable model, types of cell death, dose and time dependence dose- and time-dependent manner was evident, whereas senescence was slightly increased in response to dose but not to time. As for the 'other types of cell death' mode on day 7 showed a higher probability than the one on day 2 and as well as a prominent dose-dependence. The RCR model fits better to the experimental data than the LQ model. On day 2 there is a slight increase of the apoptotic and senescent probability with dose. On the other hand, on day 7 the shape of the curve of apoptosis differs and a sigmoidal increase with dose is observed. At both time-points, the present model fits the data reasonably well. Due to the fact that the clonogenic survival does not coincide with the one extracted from the fluorescence microscopy, a more accurate way to quantify cell death needs to be used, e.g. computerized video time-lapse (CVTL).

\section{Introduction}

Experimental and theoretical studies in radiation biology contribute to the development of radiotherapy at many levels. First of all, radiation biology offers a fundamental basis in the understanding of how radiotherapy works. It identifies the mechanisms and processes that determine the tumor and normal tissue response to irradiation and makes it possibly to understand and explain the observed phenomena. Irradiation may cause different types of damage to the cell. The damage can be lethal in the case the cells are triggered to divide during the resting period.

As a cell survival curve we can define the relationship between the radiation dose and the proportion of cells that survive. The term 'cell survival' or vice versa cell death may have a different meaning in different circumstances. For differentiated cells that do not proliferate such as, nerve or muscle cells death can be defined as the loss of a specific function. Whereas for proliferating cells such as, cells' growing in culture irreversible loss of reproductive capacity is the appropriate definition (1).

This definition has particular relevance to radiotherapy due to the fact that a tumor is locally controlled when all its cells have lost their ability to proliferate indefinitely and thus cause further growth or distant metastasis. In general the cells may go 
into different types of cell death such us death while attempting to divide which is called mitotic death. Other equally important types are apoptosis, necrosis and senescence.

Other types of cell death that are considered important are mitotic catastrophe and autophagy. Mitotic catastrophe is a cell death mode occurring either during or shortly after a failed mitosis and can be accompanied by morphological alterations including micronucleation and multinucleation. However, there is no broad consensus on the use of this term and mitotic catastrophe can lead either to apoptotic morphology or to necrosis. When autophagy is occurring in the cell, we observe lack of chromatin condensation, massive vacuolization of the cytoplasm, accumulation of autophagic vacuoles and little or no uptake by phagocytic cells in vivo. On the other hand there is no doubt that autophagy promotes cell survival, in multiple physiological and experimental settings (2). Significantly some authors claim that cells presenting features of autophagic cell death can still recover upon withdrawal of the death inducing stimulus (3).

In the present study, a mathematical model is fitted to the dose- and time-response of cell death seen in the experiments. Also a comparison of cell survival from the clonogenic assay with the cell death data from the microscopy analysis is made.

\section{Materials and methods}

Cells and culturing process. A human small-cell lung cancer (SCLC) cell line U1690 with a mutated p53 gene was used (established by Dr Jonas Berg) (4,5). These cells were maintained in monolayer cultures in Eagle's minimum essential medium (MEM with Earle's salts, Flow Laboratories, Rickmansworth, UK) containing $10 \%$ (v/v) fetal bovine serum, $100 \mathrm{IE} / \mathrm{ml}$ penicillin, $100 \mu \mathrm{g} / \mathrm{ml}$ streptomycin and $2.5 \mu \mathrm{g} / \mathrm{ml}$ fungizone (Gibco, Invitrogen, Inchinnan, UK), and incubated at $37^{\circ} \mathrm{C}, 5 \% \mathrm{CO}_{2}$ and $80 \%$ humidity in culture flasks (Costar, Badhoevedorp, The Netherlands). Cells in exponential growth phase were used in all experiments and the doubling time was approximately $24 \mathrm{~h}$.

Irradiation process. Cell suspensions were irradiated in plastic vials (Costar, 15 -ml tubes) with a ${ }^{137} \mathrm{Cs}$ irradiation chamber (Scanditronix IC 900, Instrument Scanditronix AB, Uppsala, Sweden) at Cancer Center Karolinska (Stockholm, Sweden), at a photon dose-rate of $0.5 \mathrm{~Gy} \mathrm{~min}^{-1}$ and LET of $<1 \mathrm{eV} \mathrm{nm}^{-1}$. The doses used were in the range 0-8 Gy.

Clonogenic cell survival. Standard colony formation assay was performed immediately after exposure to a ${ }^{137} \mathrm{Cs}$ source (photons). A specific number of cells were plated in Petri dishes (6-cm diameter, Costar), three replicate dishes were employed and three independent experiments were repeated for each survival point and colonies containing more than 50 cells were scored as survivors (6). Colonies obtained after 2 weeks with a change of medium after 7 days, were rinsed with phosphate-buffered saline (PBS), fixed with $8 \%$ formaldehyde solution for $5 \mathrm{~min}$ and stained with $25 \%$ Giemsa (Gurr, BDH, Pool, UK) solution for at least $15 \mathrm{~min}$. The surviving fraction (SF) was calculated as the ratio of the mean plating efficiency (PE) of irradiated cells over the PE of non-irradiated cells. PE stands for the ratio of the colo- nies counted to the number of plated cells. The PE for the non-irradiated cells was $57.2 \pm 8.5$.

Detection of different types of cell death (fluorescence microscopy). A solution of PBS mixed with acridine orange (AO; staining all parts of the cell) and Hoechst 33324 (HO; staining only DNA) (both Sigma, St. Louis, MO, USA) was used as a staining dye. The concentrations of the dyes were chosen with respect to the number of cells and the volume of the sample. The amounts used were $2.5 \mu \mathrm{l}$ of $\mathrm{HO}$ (stock $5 \mathrm{mg} / \mathrm{ml}$ ) and $2.5 \mu \mathrm{l}$ of $\mathrm{AO}$ (stock $1 \mathrm{mg} / \mathrm{ml}$ ) that were mixed with $995 \mu \mathrm{l}$ of PBS. To each cell sample 2-4 $\mu 1$ from the dye mixture was added. This concentration of the dyes gives a good amount of fluorescence light, not too bright or too weak.

Analyses were performed at a magnification, x500 in a fluorescence microscope (Leitz, Wetzlar, Germany) equipped with different filter blocks. A filter block that is consisted of an excitation light filter with high transmission between 340 and $380 \mathrm{~nm}$, a beam-splitting mirror with the edge at $400 \mathrm{~nm}$ and a barrier filter with the edge at $430 \mathrm{~nm}$ were applied for studies with $\mathrm{HO}$ and a filter block that is consisted of an excitation light filter with high transmission between 450 and $490 \mathrm{~nm}$, a beamsplitting mirror with the edge at $510 \mathrm{~nm}$ and a barrier filter with the edge at $515 \mathrm{~nm}$ were applied for studies with AO. For each sample at least 500 cells were scored and the classification of cells included normal, apoptotic /apoptotic bodies and other abnormal cells such as necrotic, necrotic/apoptotic, micronucleated and giant cells. Some indicative pictures of various modes of cell death are shown in Fig. 1.

$\beta$-galactosidase assay. Cells were washed in PBS, fixed overnight (room temperature) in $2 \%$ formaldehyde, washed twice with PBS without magnesium and calcium and incubated at $37^{\circ} \mathrm{C}$ overnight (no $\mathrm{CO}_{2}$ ) with fresh senescence-associated $\beta$-galactosidase (SA- $\beta$-Gal) stain solution at $\mathrm{pH} 6.0: 1 \mathrm{mg}$ of 5-bromo-4-chloro-3-indolyl $\beta$-D-galactosidase (X-Gal) per $\mathrm{ml}$ (stock solution $=20 \mathrm{mg}$ of potassium ferrocyanide $/ 5 \mathrm{mM}$ potassium ferricyanide/150 $\mathrm{mM} \mathrm{NaCl} / 2 \mathrm{mM} \mathrm{MgCl}_{2}$. The staining was evident after 18-24 h of incubation, and then we washed the cells twice with water before counting them in an ordinary light microscope (7). From each sample 500 cells were counted and two groups extracted, these are senescent cells and cells that may be normal or abnormal (e.g. apoptotic, necrotic, giant, etc). The cells that went into senescence incorporated the staining solution and most of them had characteristics of giant cells (Fig. 2).

\section{Mathematical modeling}

The linear quadratic (LQ) model. The LQ model has been established as the most popular model used to describe the cell survival curve assuming that there are two components related to cell kill by radiation and it is expressed by:

$$
S(D)=e^{-\alpha d-\beta d^{2}}
$$

where $S(D)$ is the fraction of cells surviving a dose $D, \alpha$ is a constant describing the initial slope of the survival curve and $\beta$ is a usually smaller constant describing the quadratic component of cell killing (8). 


\section{HO 33324 AO}

a)

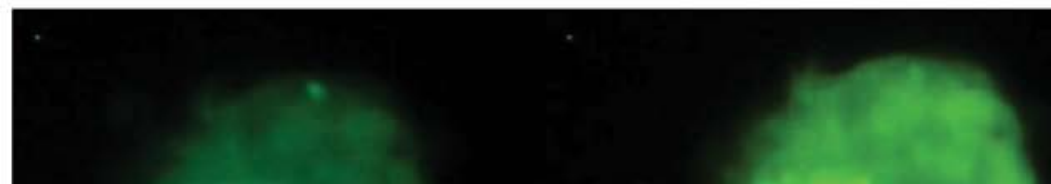

b)

c)

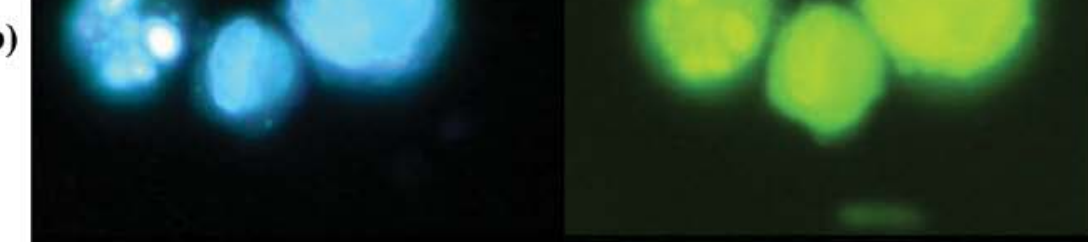

d)

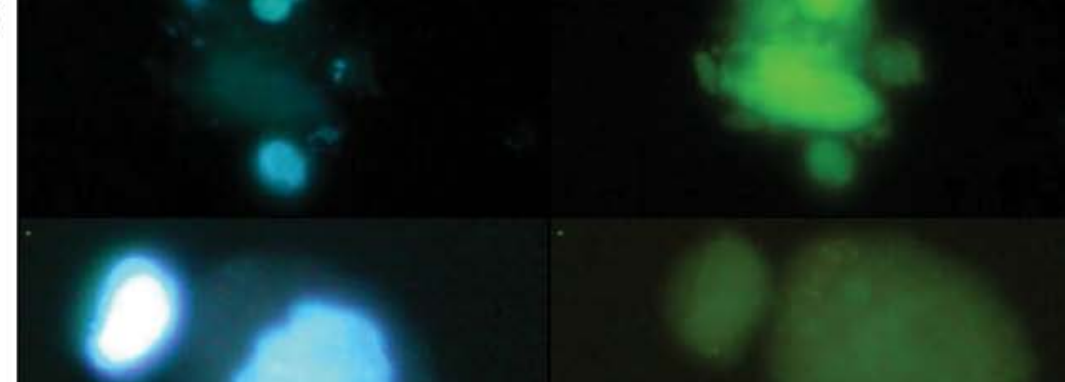

e)

Figure 1. Morphological classification of SCLC cell line (U1690) stained in a mixture of acridine orange (AO) and Hoechst (HO). (a) Necrotic-apoptotic cell; (b) apoptotic cell with pyknotic bodies and normal cells at the same field; (c) apoptotic cell (upper left), empty cell (middle), normal cells; (d) normal cell (left), giant cell (right); (e) apoptotic cell (left), normal cell (right).

The repairable-conditionally repairable $(R C R)$ model. The RCR model subdivides the total amount of damage into two distinct poisson processes: i) potentially repairable, which may also be lethal (i.e. if unrepaired or misrepaired); and ii) conditionally repairable, which may be repaired or may lead to apoptosis and other types of cell death if it has not been repaired correctly. This cell survival expression has the ability to fit most experimental data well at low doses (initial hypersensitive range), intermediate range (on the shoulder of the survival curve) and high doses (on the quasi-exponential region of the survival 


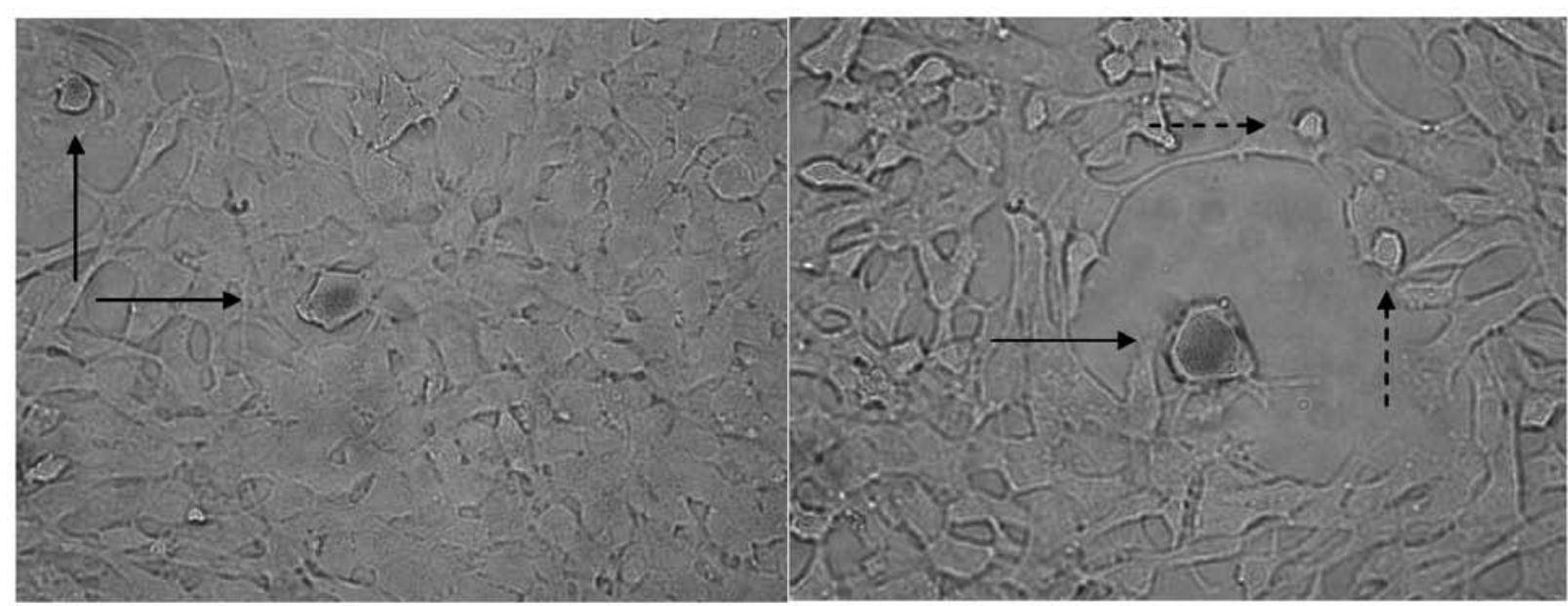

Figure 2. Cells stained with senescence associated- $\beta$-galactosidase solution. An uptake of the staining solution from the senescent cells is visible (line), dashed lines indicate normal cells.

curve). The formula of this model can be well approximated by a bi-exponential cell survival expression:

$$
S(D)=e^{-a D}+b D e^{-c D}
$$

where $D$ denotes the dose and $\alpha, b$ and $c$ are the parameters of the model. The first term $e^{-a D}$, corresponds to the fraction of cells that have not been damaged and the parameter $\alpha$ represents the initial total number of damage events per unit dose. The second term $b D e^{-c D}$, may be regarded as an expression describing the probability that a potentially repairable event proportional to $b D$ is actually repaired considering higher orders of event multiplicity. The parameter $b$ describes the maximum amount of damage that can be repaired per unit dose (9).

Cells exposed to ionizing radiation can undergo different types of cell death or repair stage. In the current study, a simplified mathematical model is introduced for describing apoptosis, senescence and other types of cell death all included in one category (necrosis, giant cells, mitotic catastrophe, micronuclei etc).

The induction of cell death is assumed to be described by poisson statistics. The probability for a cell to be alive in generation $i$ is given by the expression:

$$
P_{V}^{N}=\prod_{i=1}^{N} P_{V}(i)
$$

and it is defined as the product of probabilities in each generation for $i=1$ up to $N$. Moreover, the expected number of viable cells after $N$ divisions is given by the equation:

$$
M_{V}^{N}=2^{N} \prod_{i=1}^{N} P_{V}(i)
$$

which, corresponds to the probability $P_{V}^{N}$ multiplied by $2^{N}$. The probability for a cell to go into apoptosis after a dose
$D$ and a specific number of mitoses can be described by the formula:

$$
P_{\mathrm{A}}(D, t)=A(D) e^{-\frac{\ln 2 t}{T_{1 / 2}}}
$$

The same mathematical expression was used for the other two types of cell death.

One of the major problems that were faced, during the development of the model, was the determination of a curve, which could describe the induction of different types of cell death as a function of dose. Taking into account previous studies about determination of dose-response curves for apoptosis and the experimental data of this study, it can be assumed that the shape of such a dose-response curve can be phenomenologically described by the equation:

$$
A(D)=F_{A}\left(1-e^{-\xi_{a} D}\right)
$$

where $D$ is the dose, $\xi_{\alpha}$ is the coefficient for radiation inactivation via apoptosis, and $F_{A}$ is the maximum achievable fraction of apoptotic cells, i.e. the subpopulation, which is susceptible to radiation-induced apoptosis (10-12). The general trend followed by the curves is that there is an increase of the induction of apoptosis as a function of dose but after the intermediate dose region the curve starts bending until it reaches a plateau. The dose response-curves for senescence and other kinds of cell death are characterized by the same pattern and are given by the expressions:

$$
\begin{aligned}
& S(D)=F_{S}\left(1-e^{-\xi_{s} D}\right) \\
& O(D)=F_{o}\left(1-e^{-\xi_{o} D}\right)
\end{aligned}
$$

Also, it is assumed that the maximum achievable fraction of normal cells is equal to $F_{V}=1-F_{A}-F_{S}-F_{O}$ which means that even at 
very high doses and on day 2 cells are still viable. This assumption seems to be logical, since on day 2 after irradiation the cells have divided 1-2 times and the death process for most of them is not triggered or the death mode cannot be specified through morphological characteristics.

The comparison of the data referring to the 2 nd and 7 th day after irradiation regarding the induction of different types of cell death as a function of dose showed that the Eqs. [6], [7] and [8] had to be slightly changed. Therefore, taking into consideration the experimental data of the 7th day, the Eqs. [6-8] are modified to the following ones:

$$
\begin{aligned}
& A(D)=F_{A}\left(1-e^{-\xi_{a} D}\right)^{m_{a}} \\
& S(D)=F_{S}\left(1-e^{-\xi_{s} D}\right)^{m_{s}} \\
& O(D)=F_{O}\left(1-e^{-\xi_{o} D}\right)^{m_{o}}
\end{aligned}
$$

As can be seen, the only difference is a new variable $m$ $\left(m_{a}, m_{s}, m_{o} \neq 1\right)$. The system of Eqs. [9], [10] and [11] having one free $\left(F_{O}\right)$ and eight $\left(F_{A}, F_{S}, \xi_{\alpha}, \xi_{s}, \xi_{o}, m_{\alpha}, m_{s}\right.$ and $\left.m_{o}\right)$ dependent variables was solved by fitting it to the experimental data. It was assumed that $F_{A}+F_{S}+F_{O}=1$ thus, the free variable was calculated by the expression $F_{O}=1-F_{S}-F_{A}$. Therefore, for high doses and on day 7 after irradiation it is assumed that $F_{V}$ is negligible. Since the fate of each cell was known, next step was the extraction of an equation that could calculate the amount of normal cells or those undergoing apoptotic, senescent and other types of cell death in each generation. This equation is represented by the following expression:

$$
N_{a}^{n}=2^{n} P_{a}(n) \prod_{i-1}^{n-1} P_{v}(i)
$$

which can be interpreted as the expected number of cells going into apoptosis in generation $i$.

\section{Results}

Clonogenic cell survival. The clonogenic cell survival for p53-mutated U1690 following exposure from a ${ }^{137} \mathrm{Cs}$ source, are shown in Fig. 3. The cell survival models fitted to the experimental data were the linear quadratic (LQ) and repairable-conditionally repairable (RCR) model (Table I).

The determination of the model parameters was done by fitting the radiobiological model to the clonogenic data. In this case the fitting method that was implemented was the maximum likelihood estimation. Table II shows the values of the parameters for each model with their errors that represent the $95 \%$ confidence interval.

The goodness of fit of the models and their parameters was evaluated by Pearson's $\chi^{2}$ test (13). Although the $\chi^{2}$-value is referred as a measure of goodness of fit, conversely represents a measure of lack of fit and it should be as low as possible. Consequently the smaller the $\chi^{2}$ is the better overall fit is achieved with experimental data. However, due to the fact that the $\chi^{2}$ is dependent on the degrees of freedom, the reduced $\chi^{2}$ was included in the statistical analysis which stands for the $\chi^{2}$-value divided by the DF (number of data

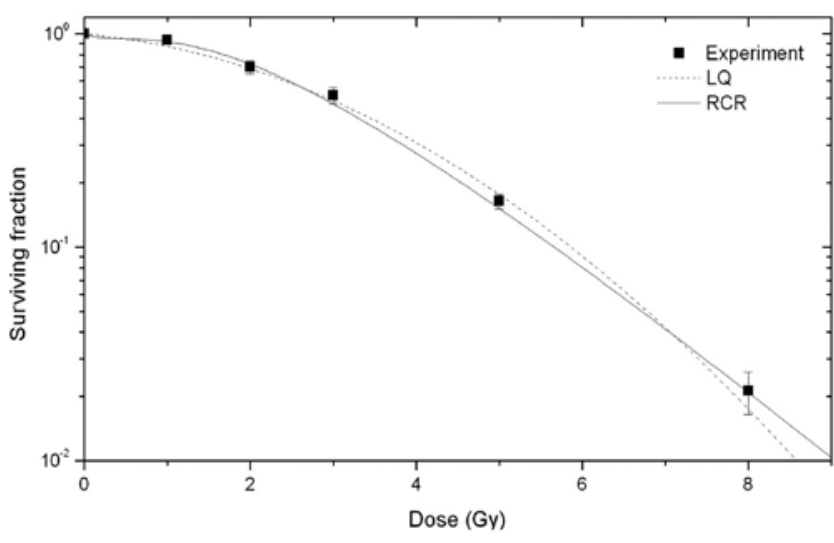

Figure 3. Clonogenic survival of the human small cell lung cancer cell line U1690 after exposure to radiation from ${ }^{137} \mathrm{Cs}$. The LQ (dashed line) and RCR (solid line) models were fitted to the experimental cell survival data. The experimental values are presented in the form of mean \pm Sem and are based on three independent experiments.

Table I. Comparison of the mean survival fraction (SF) from the experimental data with the predictions of the LQ and RCR radiobiological models.

\begin{tabular}{ccccc}
\hline Dose (Gy) & Mean & Sem & LQ & RCR \\
\hline 0 & 1.00 & 0.07 & 1.00 & 1.00 \\
1 & 0.93 & 0.03 & 0.87 & 0.92 \\
2 & 0.70 & 0.05 & 0.69 & 0.72 \\
3 & 0.52 & 0.04 & 0.49 & 0.47 \\
5 & 0.16 & 0.01 & 0.18 & 0.15 \\
8 & 0.02 & 0.00 & 0.02 & 0.02 \\
\hline
\end{tabular}

Table II. The parameter values of the LQ and RCR models, which were calculated from the experimental data using the maximum likelihood method.

\begin{tabular}{lccc}
\hline \multicolumn{2}{c}{ LQ } & \multicolumn{2}{c}{ RCR } \\
\hline & 0.0813 & & 2.128 \\
$\alpha / \mathrm{Gy}^{-1}$ & $(0.0746-0.0881)$ & $\mathrm{a} / \mathrm{Gy}^{-1}$ & $(1.963-2.365)$ \\
& 0.0531 & & 1.815 \\
$\beta / \mathrm{Gy}^{-2}$ & $(0.0519-0.0544)$ & $\mathrm{b} / \mathrm{Gy}^{-1}$ & $(1.780-1.849)$ \\
& & & 0.8182 \\
& & $\mathrm{c} / \mathrm{Gy}^{-1}$ & $(0.8116-0.8249)$ \\
LL & $-6,792.27$ & & $-6,786.98$ \\
$\chi^{2}$-value & 9.21 & & 6.10 \\
Reduced & & & \\
$\chi^{2}$-value & 0.576 & & 0.407 \\
AIC & $13,588.5$ & & $13,580.0$ \\
\hline
\end{tabular}

The uncertainties of the model parameters are expressed in terms of their $95 \%$ confidence intervals. The $\chi^{2}$, the reduced $\chi^{2}$ and the Akaike's Information Criterion (AIC)-values are also presented.

points reduced by the number of parameters in the respective model) (14). In the present study the $\chi^{2}$ and reduced $\chi^{2}$-values 


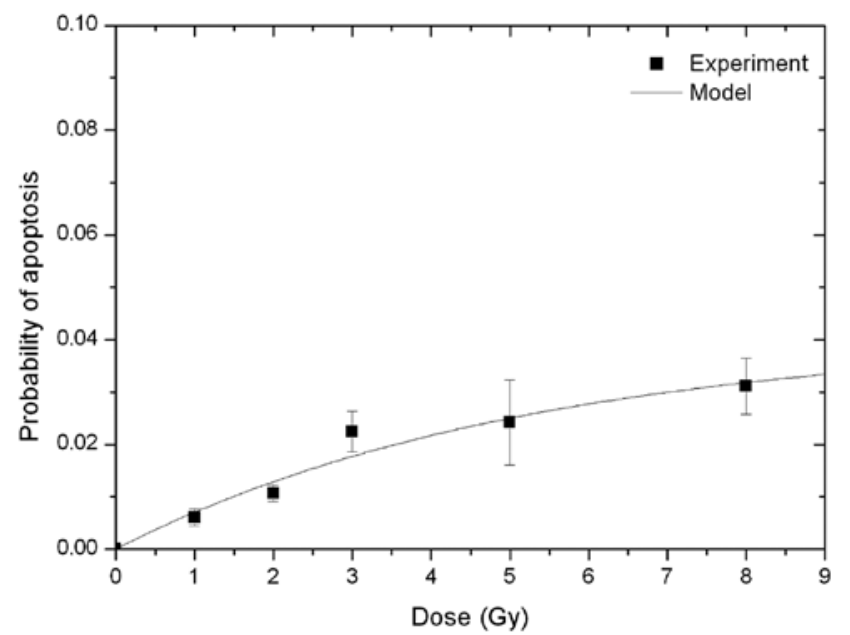

Table III. Experimental and estimated probability of the induction of cell death (apoptosis, senescence and other abnormal cells) $48 \mathrm{~h}$ after irradiation.

\begin{tabular}{ccccc}
\hline $\begin{array}{c}\text { Day 2 } \\
\text { Dose (Gy) }\end{array}$ & Experiment & $\begin{array}{c}\text { Apoptosis } \\
\text { Sem }\end{array}$ & Model & MLE \\
\hline 0 & 0.000 & 0.000 & 0.000 & 0.000 \\
1 & 0.006 & 0.002 & 0.007 & -0.037 \\
2 & 0.011 & 0.002 & 0.013 & -0.059 \\
3 & 0.224 & 0.004 & 0.018 & -0.108 \\
5 & 0.024 & 0.008 & 0.025 & -0.114 \\
8 & 0.031 & 0.005 & 0.032 & -0.139 \\
& & & Sum & -0.457 \\
$\mathrm{~F}_{\mathrm{A}}$ & 0.041 & & & \\
$\xi_{\alpha}$ & 0.191 & & & \\
\hline Day 2 & & Senescence & & \\
Dose $(\mathrm{Gy})$ & Experiment & Sem & Model & MLE \\
\hline 0 & 0.000 & 0.000 & 0.000 & 0.000 \\
1 & 0.011 & 0.003 & 0.008 & -0.060 \\
2 & 0.015 & 0.005 & 0.015 & -0.079 \\
3 & 0.189 & 0.005 & 0.020 & -0.093 \\
5 & 0.025 & 0.005 & 0.028 & -0.118 \\
8 & 0.037 & 0.007 & 0.034 & -0.157 \\
& & & Sum & -0.509 \\
$\mathrm{~F}_{\mathrm{S}}$ & 0.042 & & & \\
$\xi_{\mathrm{s}}$ & 0.220 & & & \\
\hline & & & & \\
\hline & & & & \\
\hline
\end{tabular}

\begin{tabular}{ccccc}
\hline \multirow{2}{*}{$\begin{array}{c}\text { Day } 2 \\
\text { Dose (Gy) }\end{array}$} & \multicolumn{4}{c}{ Other types of cell death } \\
& Experiment & Sem & Model & MLE \\
\hline 0 & 0.000 & 0.000 & 0.000 & 0.000 \\
1 & 0.007 & 0.002 & 0.008 & -0.043 \\
2 & 0.010 & 0.002 & 0.014 & -0.058 \\
3 & 0.026 & 0.002 & 0.021 & -0.120 \\
5 & 0.032 & 0.004 & 0.031 & -0.142 \\
8 & 0.040 & 0.005 & 0.042 & -0.169 \\
& & & Sum & -0.533 \\
$F_{\mathrm{O}}$ & 0.067 & & & \\
$\xi_{\mathrm{o}}$ & 0.122 & & & \\
\hline
\end{tabular}

The values in the second column represent the mean \pm Sem of three independent experiments performed in triplicate.

Figure 4. Dose-dependence of the apoptotic (upper diagram), senescent (middle diagram) and other types of cell death (lower diagram) probability evaluated at $48 \mathrm{~h}$ after irradiation.

for the RCR model are less than the one for the LQ, so we can claim that the RCR model is fitted better to the experimental data. In addition, another way of measuring the goodness of fit of the models is by means of the Akaike's Information Criterion (AIC). The radiobiological model with the smallest AIC-value represents the one that it is fitted better to the data, and as shown in Table II, it is the RCR model.

Mathematical modeling of the dose- and time-response of cell death and comparison to the experimental data. The dose-dependence, of different types of cell death, evaluated at 2 days after irradiation is given in Fig. 4 where it seems that after the initial increase of the probability in the three graphs, the steepness of the curve becomes lower, which gradually leads to saturation. Thus, the selection of the mathematical model that is described by the Eqs. [6], [7] and [8] estimates fairly well the results acquired by the experiments (Table III). Furthermore, the maximum likelihood estimation (MLE) was used to determine the best estimates of the model parameters by maximizing the likelihood to reproduce the given pattern of observations. 

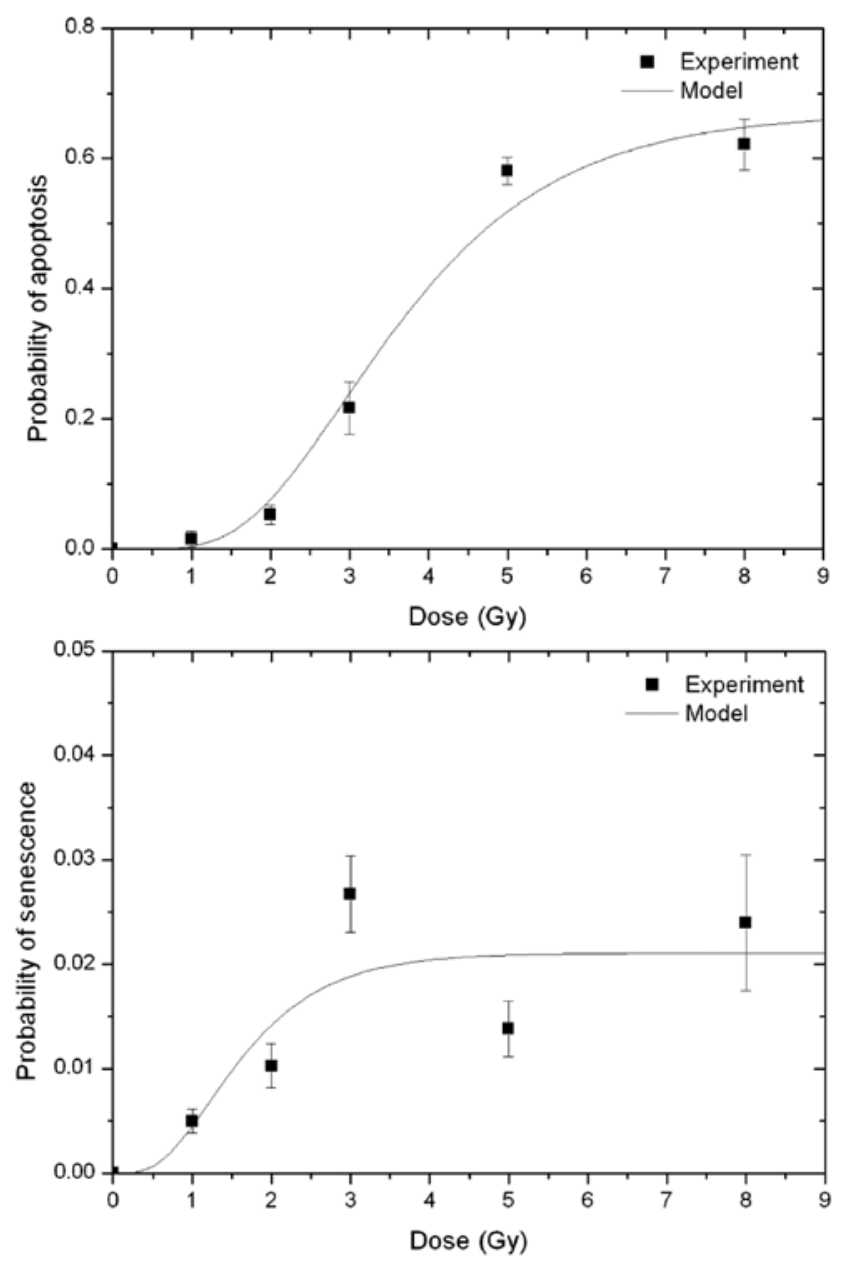

Table IV. Experimental and estimated probability of the induction of cell death (apoptosis, senescence and other abnormal cells) 7 days after irradiation.

\begin{tabular}{|c|c|c|c|c|}
\hline \multirow{2}{*}{$\begin{array}{c}\text { Day } 7 \\
\text { Dose (Gy) }\end{array}$} & \multicolumn{4}{|c|}{ Apoptosis } \\
\hline & Experiment & Sem & Model & MLE \\
\hline 0 & 0.000 & 0.000 & 0.000 & 0.000 \\
\hline 1 & 0.015 & 0.012 & 0.004 & -0.088 \\
\hline 2 & 0.053 & 0.015 & 0.077 & -0.211 \\
\hline 3 & 0.217 & 0.040 & 0.239 & -0.525 \\
\hline 5 & 0.581 & 0.021 & 0.518 & -0.688 \\
\hline \multirow[t]{2}{*}{8} & 0.621 & 0.039 & 0.648 & -0.665 \\
\hline & & & Sum & -2.176 \\
\hline $\mathrm{F}_{\mathrm{A}}$ & 0.671 & & & \\
\hline$\xi_{\alpha}$ & 0.666 & & & \\
\hline $\mathrm{m}_{\alpha}$ & 7.065 & & & \\
\hline Day 7 & \multicolumn{3}{|c|}{ Senescence } & \\
\hline Dose (Gy) & Experiment & Sem & Model & MLE \\
\hline 0 & 0.000 & 0.000 & 0.000 & 0.000 \\
\hline 1 & 0.005 & 0.001 & 0.005 & -0.031 \\
\hline 2 & 0.010 & 0.002 & 0.014 & -0.058 \\
\hline 3 & 0.027 & 0.004 & 0.019 & -0.125 \\
\hline 5 & 0.014 & 0.003 & 0.021 & -0.074 \\
\hline \multirow[t]{2}{*}{8} & 0.024 & 0.006 & 0.021 & -0.113 \\
\hline & & & Sum & -0.401 \\
\hline $\mathrm{F}_{\mathrm{S}}$ & 0.021 & & & \\
\hline$\xi_{\mathrm{s}}$ & 1.235 & & & \\
\hline $\mathrm{m}_{\mathrm{s}}$ & 4.489 & & & \\
\hline
\end{tabular}

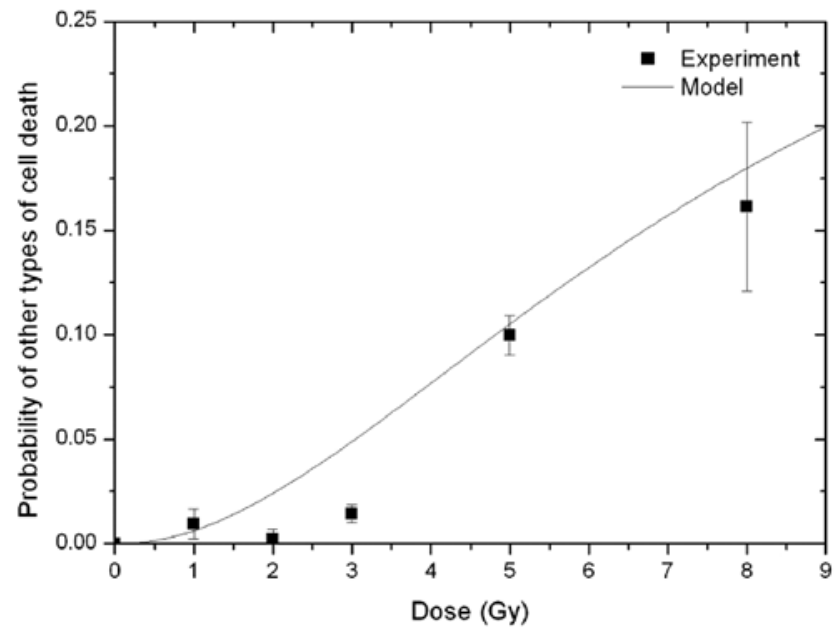

Day 7

Other types of cell death

Dose (Gy) Experiment Sem Model MLE

\begin{tabular}{lllll}
\hline 0 & 0.000 & 0.000 & 0.000 & 0.000 \\
1 & 0.014 & 0.007 & 0.006 & -0.079 \\
2 & 0.012 & 0.005 & 0.024 & -0.070 \\
3 & 0.041 & 0.004 & 0.049 & -0.172 \\
5 & 0.114 & 0.009 & 0.105 & -0.354 \\
8 & 0.185 & 0.040 & 0.180 & -0.480 \\
& & & Sum & -1.156
\end{tabular}

$\begin{array}{lllll}\mathrm{F}_{\mathrm{O}} & 0.308 & & & \\ \xi_{\mathrm{o}} & 0.192 & & \\ \mathrm{~m}_{\mathrm{o}} & 2.232 & \text { Sum } & -3.733\end{array}$

Figure 5. Dose-dependence of the apoptotic, senescent and other types of cell death probability evaluated at 7 days after irradiation. Details as in Fig. 4.

The values in the second column represent the mean \pm Sem of three independent experiments performed in triplicate.

Alternatively to the parameter interpretation mentioned above, it can be stated that the constants $F_{A}, F_{S}, F_{O}$ stand for the maximum achievable apoptotic, senescent and other type of cell death probability, respectively. Whereas, $\xi_{\alpha}, \xi_{s}, \xi_{o}$ represent coefficients for radiation inactivation via apoptosis, senescence and other types of cell death.

The quantification of cell death 2 days after exposure shows that the maximum values have been reached after a dose of $8 \mathrm{~Gy}$ or that they represent more than the $70 \%$ of the maximum

value $\left(F_{A}, F_{S}\right.$ or $\left.F_{O}\right)$. Thus, it was of great significance to follow the cells further in time so as to investigate whether the curves exhibit a similar trend or whether there is a change in the shape of the dose-response (cell death) curves with time.

The examination of different types of cell death 7 days after irradiation demonstrated a significant induction of apoptosis in a dose- and time-dependent manner, whereas the dependence of senescence with dose was slightly increased, but not with time (see Figs. 4 and 5). The 3rd category 'other abnormal cells' 


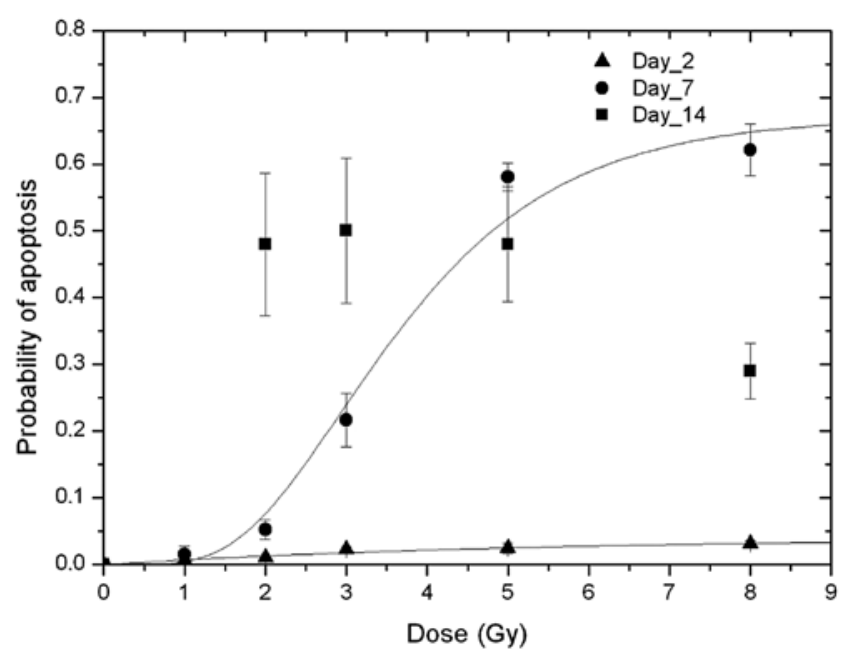

Figure 6. Apoptotic probability as a function of dose $(0,1,2,3,5$ and 8 Gy) and time (2, 7 and 14 days).

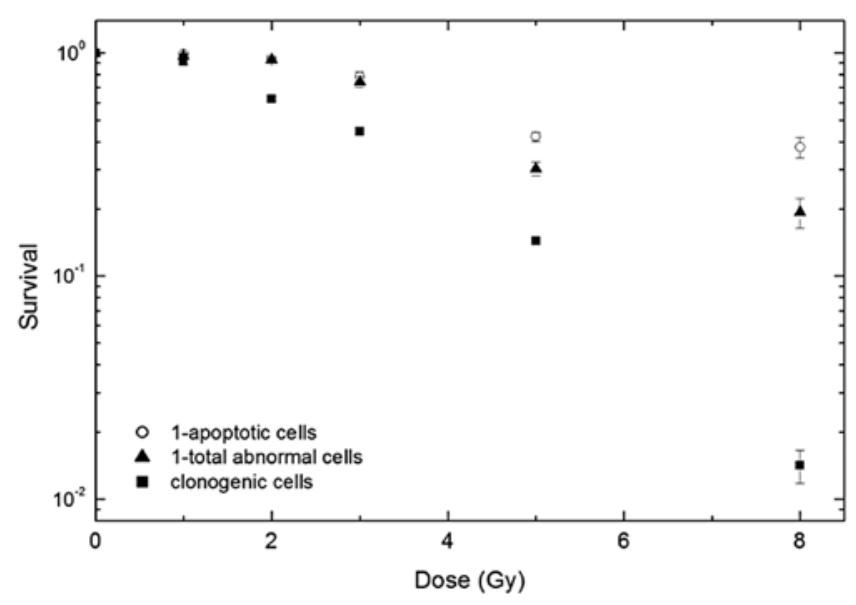

Figure 7. Comparison of the clonogenic survival data with the fluorescence microscopy data (day 14) as a function to dose. There is a close correlation for 0 up to $3 \mathrm{~Gy}$ and then at 5 and $8 \mathrm{~Gy}$ a large discrepancy is noted. Squares, clonogenic cells; circles, 1-apoptotic cells; triangles, 1-total abnormal cells.

in the 7th day showed a higher probability than the one in the 2nd day as well as prominent dose-dependence. It is obvious that the 7 day curves do not follow a similar shape compared to the 2 day curves. At this point, a more sophisticated expression had to be introduced that incorporates a third variable which gives to the curves a sigmoid shape. The fitting of the model to the experimental data was done by means of maximum likelihood estimation (see Table IV). By combining these data with those from the fluorescence microscopy, a trigger of a specific type of cell death such as apoptosis can be identified (Fig. 6).

\section{Discussion}

The modeling of cell death on day 14 was not possible to perform due to problems faced with the protocol of the senescence assay and as a result with the quantification of the senescent cells. Thus, in Fig. 7, the apoptotic probability is presented as a function of dose for 3 different time-points as well as the modeling of the experimental data referring to the 2 nd and 7 th day after irradiation. As is shown, on day 2 there is a slight increase of the apoptotic probability with dose, whereas on day 7 the shape of the curve differs and a sigmoidal increase with dose is observed. The decrease of apoptosis on the 14th day compared to the 7th day at 5 and 8 Gy can most likely be described by several factors, such as triggering of other types of cell death, disintegration and disappearance of the cells after irradiation with 5 and 8 Gy. Moreover, the large error bars characterizing the data of the 14th day can be justified by the significant variations between the 3 experiments.

At both time-points ( 2 and 7 days) the model fits the experimental data quite well. It may seem as if in the 7 th day the total number of parameters in the model differ in comparison to those on the 2 nd day but this is misleading because on day 2 it is assumed that the constants $m_{a}, m_{\xi}, m_{o}$ are equal to 1 .

Few studies have been published on the investigation of different types of cell death after irradiation as a function to dose and time. One shows that photons induced apoptosis in a human melanoma cell line AA, which increased in a dosedependent manner up to $2 \mathrm{~Gy}$ and at $48 \mathrm{~h}$. Beyond that point, there was no major increase in apoptosis with dose. Thus, it appears that the shape of the curve is consistent with the one in this study. Also, the induction of apoptosis was evident at $72 \mathrm{~h}$ (11). Moreover, in another study where the death pattern of a human lung cancer cell line (H1299) with a mutated p53 was analyzed after irradiation with X-rays, it was indicated that the percentage of apoptosis at 2 Gy and 2 days is in close correlation with the presented experimental data (15). However, in both studies the cells were followed only up to $72 \mathrm{~h}$, consequently we can not compare later time-points.

None of any previous cellular senescence assays have investigated this specific cell line (U1690) but based on an extended study in accelerated senescence among seven different cell lines with a wild-type p53 irradiated with a ${ }^{60} \mathrm{Co}$ source, two of them (renal carcinoma and malignant melanoma) demonstrated $<2 \%$ senescence after 4 Gy and $\sim 10 \%$ after 8 Gy on the 6th day after irradiation. At least the dose point of $4 \mathrm{~Gy}$ is in agreement with the one in the present study (16). In a more recent article, the intensity of cellular staining for SA- $\beta$-Gal increased in a time- and dose-dependent manner in a head and neck squamous cell carcinoma cell line with a mutated p53. However, a further investigation whether an enhanced SA- $\beta$-Gal activity is related to a senescence-like mechanism or not showed no accumulation of p16 and p21 proteins. Thus, the enhanced activity of SA- $\beta$-Gal seems to be the result of cellular stress rather than of an irreversible senescence-like process $(17,18)$.

For cells that go into apoptosis shortly after irradiation without dividing, such as lymphocytes and lymphomas, a reasonably accurate assessment of the amount of apoptosis should be possible by quantifying apoptosis in the irradiated population as a whole. But in the case of post-mitotic death, individual cells and their progeny must be followed for several generations after irradiation. A major problem in studies that aim to quantify different types of cell death in relation to time relies on the fact that there is no distinction between proliferating clonogenic cells and non-clonogenic descendants. Furthermore, apoptosis and/or senescence are occurring in non-clonogenic descendants during several days and generations after irradiation. 
As a consequence, in our study since it is assumed that all cells are clonogenic, the percentage of apoptosis and senescence will be underestimated. Another issue that could not be neglected is the disintegration and disappearance of cells after 5 or $8 \mathrm{~Gy}$ at late time-points. Due to this fact, the fluorescence microscopy data will not coincide with those from the clonogenic survival. A potential solution could be the use of computerized video time-lapse microscopy in which individual cells are followed and the mode of cell death can be determined. Moreover, in this way a more accurate quantification of apoptosis and other types of cell death can be assessed $(19,20)$. However, this method has also limitations since the narrow field of view and the limited number of cells constitutes an actual drawback.

Regarding the clonogenic assay it seems that the RCR model fits better the experimental data compared to the LQ model. The quantification of cell death by means of morphological features and the senescence assay showed that on day 2 after irradiation there is a slight increase of the apoptotic and senescent probability with dose. On the other hand, on day 7 after irradiation, the shape of the curve of apoptosis differs and a sigmoidal increase with dose is observed. At both time points the mathematical model fits the experimental data reasonable well. Finally, due to the fact that the clonogenic survival does not coincide with the one extracted using fluorescence microscopy, a more accurate way of quantification of cell death need to be used, eventually such as the computerized video time-lapse microscopy.

\section{Acknowledgements}

The authors would like to thank Professor Niko Papanikolaou for his valuable contribution in the revision of the work.

\section{References}

1. Hall EJ and Giaccia AJ: Radiobiology for the Radiologist. 6th edition. Lippincott Williams \& Wilkins, Philadelphia, PA, 2005.

2. Neufeld TP and Baehrecke EH: Eating on the fly: function and regulation of autophagy during cell growth, survival and death in Drosophila. Autophagy 4: 557-562, 2008.

3. Boya P, González-Polo RA, Casares N, Perfettini JL, Dessen P, Larochette N, Métivier D, Meley D, Souquere S, Yoshimori T, Pierron G, Codogno P and Kroemer G: Inhibition of macroautophagy triggers apoptosis. Mol Cell Biol 25: 1025-1040, 2005 .

4. Bergh J, Nilsson K, Ekman R and Giovanella B: Establishment and characterization of cell lines from human small and large cell carcinomas of the lung. Acta Pathol Microbiol Immunol Scand A 93: 133-147, 1985.
5. Bergqvist M, Brattstrom D, Gullbo J, Hesselius $\mathrm{P}$, Brodin $\mathrm{O}$ and Wagenius G: p53 status and its in vitro relationship to radiosensitivity and chemosensitivity in lung cancer. Anticancer Res 23: 1207-1212, 2003

6. Puck TT and Marcus PI: Action of X-rays on mammalian cells. J Exp Med 103: 653-666, 1956.

7. Dimri GP, Lee X, Basile G, Acosta M, Scott G, Roskelley C, Medrano EE, Linskens M, Rubelj I, Pereira-Smith O, Peacocke $\mathrm{M}$ and Campisi J: A biomarker that identifies senescent human cells in culture and in aging skin in vivo. Proc Natl Acad Sci USA 26: 9363-9367, 1995.

8. Sinclair WK: The shape of radiation survival curves of mammalian cells cultured in vitro. In: Biophysical Aspects of Radiation Quality. Technical Reports Series no. 58. IAEA, Lyon, pp862-866, 1966.

9. Lind BK, Persson LM, Edgren MR, Hedlöf I and Brahme A: Repairable-conditionally repairable damage model based on dual poisson processes. Radiat Res 160: 366-375, 2003.

10. Ling $\mathrm{CC}$, Chen $\mathrm{CH}$ and Fuks Z: An equation for the dose response of radiation-induced apoptosis: possible incorporation with the LQ model. Radiother Oncol 33: 17-22, 1994.

11. Meijer AE, Jernberg AR, Heiden T, Stenerlow B, Persson LM, Tilly N, Lind BK and Edgren MR: Dose and time dependent apoptotic response in a human melanoma cell line exposed to accelerated boron ions at four different LET. Int J Radiat Biol 81: 261-272, 2005.

12. Chang $\mathrm{CH}$, Zhang $\mathrm{J}$ and Ling CC: Transfected c-myc and c-Ha-ras modulate radiation-induced apoptosis in rat embryo cells. Radiat Res 139: 307-315, 1994.

13. Baltas D and Grassman J: Volume effect and normal tissue responses. In: Modelling in Clinical Radiobiology. Baier K and Baltas D (eds). Vol. 2. Albert-Ludwigs-University, Freiburg, 1997.

14. Adamus-Gorka M: Improved dose response modelling for normal tissue damage and therapy optimization. $\mathrm{PhD}$ Thesis, University of Stockholm, Sweden, 2008.

15. Takahashi A, Matsumoto H, Yuki K, Yasumoto J-C, Kajiwara A, Aoki M, Furusawa Y, Ohnishi K and Ohnishi T: High-LET radiation enhanced apoptosis but not necrosis regardless of p53 status. Int J Radiat Oncol Biol Phys 60: 591-597, 2004.

16. Mirzayans R, Scott A, Cameron M and Murray D: Induction of accelerated senescence by gamma radiation in human solid tumor derived cell lines expressing wild-type TP53. Radiat Res 163: 53-62, 2005

17. Maalouf M, Alphonse G, Colliaux A, Beuve M, Trajkovic-Bodennec S, Battiston-Montagne P, Testard I, Chapet O, Bajard M, Taucher-Scholz G, Fournier C and Rodriguez-Lafrasse C: Different mechanisms of cell death in radiosensitive and radioresistant p53 mutated head and neck squamous cell carcinoma cell lines exposed to carbon ions and X-rays. Int J Radiat Oncol Biol Phys 74: 200-209, 2009.

18. Severino J, Allen RG, Balin S, Balin A and Cristofalo VJ: Is beta-galactosidase staining a marker of senescence in vitro and in vivo? Exp Cell Res 257: 162-171, 2000.

19. Forrester HB, Vidair CA, Albright N, Ling CC and Dewey WC: Using computerized video time lapse for quantifying cell death of X-irradiated rat embryo cells transfected with c-myc or c-Ha-ras. Cancer Res 59: 931-939, 1999.

20. Forrester HB, Albright $\mathrm{N}$, Ling $\mathrm{CC}$ and Dewey WC: Computerized video time-lapse (CVTL) analysis of apoptosis of REC: Myc cells X-irradiated in different phases of the cell cycle. Radiat Res 154: 625-639, 2000. 